\title{
Prédiction des tensions superficielles, facteurs acentriques et indices de corrélation des hydrocarbures purs
}

\author{
A. Adamou ${ }^{1}$, N. Skander ${ }^{2}$ and C.E. Chitour ${ }^{1}$ \\ 1 Laboratoire de valorisation des énergies fossiles, Département de génie chimique, \\ École nationale polytechnique, Alger - Algérie \\ 2 Centre de recherche et développement de Sonatrach, avenue du 1er-Novembre, 35000 Boumerdès - Algérie \\ e-mail: skander_nachida@yahoo.fr - lavalef@yahoo.fr
}

Résumé - Une nouvelle méthode de contribution de groupes pour l'estimation des tensions superficielles, facteurs acentriques et indices de corrélation des hydrocarbures purs a été établie. Les déviations absolues moyennes (average absolute deviations, $A A D$ ) enregistrées entre les valeurs tabulées et celles calculées sont de 2,5\%, 4,1\% et 1,5\% respectivement pour les tensions superficielles, les facteurs acentriques et les indices de corrélation.

Abstract - Prediction of Surface Tensions, Acentric Factors and Refractive Index Parameters of Pure Hydrocarbons - A new group contribution method for the estimation of surface tensions, acentric factors and refractive index parameters of pure hydrocarbons was established. The average absolute deviations $(A A D)$ obtained between tabulated and calculated values are respectively $2.5 \%, 4.1 \%$ and $1.5 \%$ for surface tension, acentric factor and refractive index parameter. 


\section{NOTATIONS}

$\begin{array}{ll}\mathrm{AAD} & \text { déviation absolue moyenne (en } \%) \\ a, b, c, d, & \text { paramètres des équations de contribution de } \\ m, n \text { et } p & \text { groupes } \\ I & \text { indice de corrélation } \\ M & \text { masse molaire (g/mole) } \\ N_{c} & \text { nombre d'atomes de carbone } \\ T_{b} & \text { température d'ébullition }(\mathrm{K}) .\end{array}$

\section{Lettres grecques}

$\theta \quad$ propriété physique donnée

$\Delta \theta_{i} \quad$ contribution du groupement $i$ à la propriété $\theta$

$\omega$ facteur acentrique

$\sigma \quad$ tension superficielle à $20^{\circ} \mathrm{C}(\mathrm{N} / \mathrm{m})$.

\section{INTRODUCTION}

La connaissance des propriétés physiques et thermodynamiques des hydrocarbures purs est nécessaire pour l'ingénieur pétrolier. Ces paramètres sont en général obtenus par le biais de méthodes expérimentales. Cependant, les limitations que présentent ces dernières, à savoir un temps de manipulation long et des équipements onéreux, font que l'ingénieur a souvent recours aux corrélations et aux méthodes graphiques pour accéder rapidement à ces paramètres. Parmi les corrélations existantes, les méthodes dites de contribution de groupes sont largement utilisées et continuent à faire l'objet de nombreux travaux. Ces méthodes ont pour objectif principal de rapporter les propriétés des corps purs à leur structure moléculaire. Leurs formes et leurs précisions sont assez variées et dépendent principalement du choix des groupements structuraux et de la relation mathématique choisie pour représenter les données expérimentales.

De nombreux auteurs se sont intéressés à ce type de méthodes pour prédire les propriétés physiques et thermodynamiques des corps purs. Parmi les travaux existants, nous pouvons citer ceux de Lydersen (1955) pour les propriétés critiques et repris par la suite par Joback (1984). Benson (1969) a proposé des corrélations de contribution de groupes pour la prédiction de la capacité calorifique et de l'entropie standard du gaz parfait. Constantinou et Gani (1994), Constantinou et al. (1995) ont établi des équations pourestimer les propriétés critiques et thermiques. Cependant, la plupart des corrélations existantes ne permettent pas de distinguer entre les différents isomères, d'une part, et ne s'appliquent pas aux composés lourds à nombres d'atomes decarbone élevés, d'autre part. Des travaux continuent à voir le jour pour tenter de remédier à ces insuffisances.

Dans un tel contexte, nous avons élaboré une nouvelle méthode de contribution de groupes pour l'estimation des tensions superficielles $\sigma$ à $20^{\circ} \mathrm{C}$, des indices de corrélations $I$ et des facteurs acentriques $\omega$ des hydrocarbures purs. La comparaison des nouvelles corrélations avec les données des tables a fourni des déviations standard moyennes de 2,5, 4,1 et $1,5 \%$ respectivement pour les tensions superficielles, les facteurs acentriques et les indices de corrélation.

Cette étude est la suite d'un premier travail (Skander et Chitour, 2002) qui a abouti à l'établissement de corrélations de contribution de groupes pour la prédiction de la densité, de la température d'ébullition et de la température de congélations des hydrocarbures purs.

\section{CORRÉLATIONS PROPOSÉES}

La démarche suivie pour l'élaboration de la nouvelle méthode a consisté dans une première étape en la collecte des données des propriétés des hydrocarbures purs. Les banques de données du DIPPR (Design Institute for Physical Property Data, 1995), développées par The Institute of Chemical Engineering de l'Université de Pennsylvanie, et de la banque TRC (Thermodynamic Table Database, 1994), développées par The Thermodynamic Research Center de l'Université de Houston au Texas, ont été utilisées pour constituer la base de données à partir de laquelle nous avons élaboré les corrélations. Dans une seconde étape, nous avons procédé à l'identification des groupements structuraux présents dans les différentes molécules de la base de données. La démarche suivie pour le développement des groupements chimiques est décrite en détail dans l'étude Skander et Chitour (2002).

Une fois les données des propriétés disponibles et les groupements chimiques bien définis, nous avons procédé à l'élaboration des corrélations de contribution de groupes. Nous avons alors testé différentes équations mathématiques découlant de la forme généralisée reliant la propriété $\theta$ et les contributions $\Delta \theta_{i}$ des groupements $i$ :

$$
F(\theta)=a+b^{*} \Sigma \Delta \theta_{i}+c^{*}\left(\Sigma \Delta \theta_{i}\right)^{m}+d^{*}\left(\Sigma \Delta \theta_{i}\right)^{n}
$$

Les vingt équations qui dérivent de cette forme généralisée sont reportées dans le tableau 1 . Le but de cette opération est de tester différentes formes mathématiques et de sélectionner ensuite celle qui fournit l'écart le plus faible par rapport aux données des tables (DIPPR, TRC) prises comme référence.

Les paramètres $a, b, c, d, m, n$ et $p$ de l'équation (1) sont des constantes évaluées par régression multilinéaire en utilisant l'algorithme de Levenberg-Marquardt. À la convergence du processus itératif, les valeurs des paramètres des différentes équations de contribution de groupes sont déterminées et leurs performances évaluées par le biais des écarts moyens enregistrés par rapport aux valeurs de la base de données, et également, par leur pouvoir d'extrapolation aux hydrocarbures lourds. 


\section{TABLEAU 1}

Les différentes formes de l'équation $F(\theta)$

The different forms of equation $F(\theta)$

\begin{tabular}{|c|c|}
\hline Équation (1) & $\theta=b^{*} \Sigma \Delta \theta_{i}$ \\
\hline Équation (2) & $\exp (\theta / p)=b^{*} \Sigma \Delta \theta_{i}$ \\
\hline Équation (3) & $(1 / \theta)^{p}=b^{*} \Sigma \Delta \theta_{i}$ \\
\hline Équation (4) & $(M / \theta)=b^{*} \Sigma \Delta \theta_{i}$ \\
\hline Équation (5) & $\left(T_{b} / \theta\right)=b^{*} \Sigma \Delta \theta_{i}$ \\
\hline Équation (6) & $\theta=a+b^{*} \Sigma \Delta \theta_{i}$ \\
\hline Équation (7) & $\exp (\theta / p)=a+b^{*} \Sigma \Delta \theta_{i}$ \\
\hline Équation (8) & $(1 / \theta)^{p}=a+b^{*} \Sigma \Delta \theta_{i}$ \\
\hline Équation (9) & $(M / \theta)=a+b^{*} \Sigma \Delta \theta_{i}$ \\
\hline Équation (10) & $\left(T_{b} / \theta\right)=a+b^{*} \Sigma \Delta \theta_{i}$ \\
\hline Équation (11) & $\theta=a+b^{*} \Sigma \Delta \theta_{i}+c\left(\Sigma \Delta \theta_{i}\right)^{m}$ \\
\hline Équation (12) & $\exp (\theta / p)=a+b^{*} \Sigma \Delta \theta_{i}+c\left(\Sigma \Delta \theta_{i}\right)^{m}$ \\
\hline Équation (13) & $(1 / \theta)^{p}=a+b^{*} \Sigma \Delta \theta_{i}+c\left(\Sigma \Delta \theta_{i}\right)^{m}$ \\
\hline Équation (14) & $(M / \theta)=a+b^{*} \Sigma \Delta \theta_{i}+c\left(\Sigma \Delta \theta_{i}\right)^{m}$ \\
\hline Équation (15) & $\left(T_{b} / \theta\right)=a+b^{*} \Sigma \Delta \theta_{i}+c\left(\Sigma \Delta \theta_{i}\right)^{m}$ \\
\hline Équation (16) & $\theta=a+b^{*} \Sigma \Delta \theta_{i}+c\left(\Sigma \Delta \theta_{i}\right)^{m}+d\left(\Sigma \Delta \theta_{i}\right)^{n}$ \\
\hline Équation (17) & $\exp (\theta / p)=a+b^{*} \Sigma \Delta \theta_{i}+c\left(\Sigma \Delta \theta_{i}\right)^{m}+d\left(\Sigma \Delta \theta_{i}\right)^{n}$ \\
\hline Équation (18) & $(1 / \theta)^{p}=a+b^{*} \Sigma \Delta \theta_{i}+c\left(\Sigma \Delta \theta_{i}\right)^{m}+d\left(\Sigma \Delta \theta_{i}\right)^{n}$ \\
\hline Équation (19) & $(M / \theta)=a+b^{*} \Sigma \Delta \theta_{i}+c\left(\Sigma \Delta \theta_{i}\right)^{m}+d\left(\Sigma \Delta \theta_{i}\right)^{n}$ \\
\hline Équation (20) & $\left(T_{b} / \theta\right)=a+b^{*} \Sigma \Delta \theta_{i}+c\left(\Sigma \Delta \theta_{i}\right)^{m}+d\left(\Sigma \Delta \theta_{i}\right)^{n}$ \\
\hline
\end{tabular}

\section{Sélection des meilleures corrélations}

Pour sélectionner les meilleures corrélations parmi les différentes équations testées, nous avons retenu dans une première phase, et ce, pour chacune des trois propriétés, les équations ayant fourni relativement de faibles écarts par rapport aux données des tables. Dans une seconde étape, nous avons appliqué aux équations présélectionnées le deuxième critère de sélection en prenant comme référence les corrélations de Riazi El Sahhaf (1995) pour l'extrapolation des corrélations donnant la tension superficielle et le facteur acentrique. Pour l'indice de corrélation, nous nous sommes basés sur la valeur limite de l'indice de réfraction préconisée par Wuithier
(1972). En procédant ainsi, nous avons pu sélectionner les équations (13), (7) et (18) respectivement pour l'estimation du facteur acentrique, de la tension superficielle et de l'indice de corrélation. Les écarts moyens enregistrés par les équations sélectionnées, et pour chaque famille d'hydrocarbures étudiée, sont reportés dans le tableau 2. Les paramètres des équations choisies pour la prédiction des trois propriétés sont donnés dans le tableau 3. Leur performance pour l'estimation des propriétés des $n$-paraffines est clairement illustrée sur les figures 1, 2 et 3 qui montrent un bon lissage des données par les équations sélectionnées. Dans le tableau 4, nous avons comparé nos corrélations avec d'autres corrélations basées sur la structure moléculaire et recommandées dans la littérature. Nous remarquons que nos équations sont aussi performantes, voire meilleures dans certains cas pour la prédiction des facteurs acentriques, des tensions superficielles et des indices de corrélation des hydrocarbures purs.

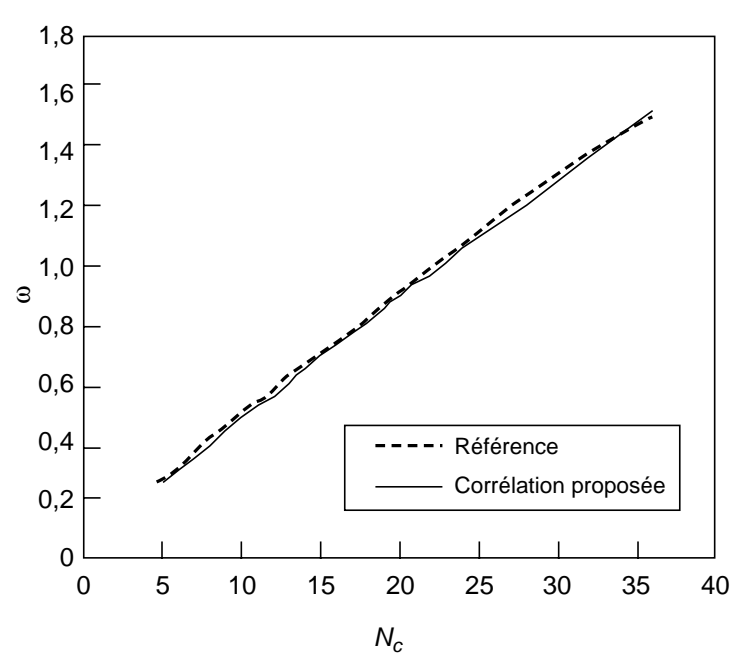

Figure 1

Facteur acentrique en fonction du nombre d'atomes de carbone des paraffines.

Acentric factors versus n-paraffin carbon atoms.

TABLEAU 2

Écarts enregistrés par les équations $F(\theta)$ pour la prédiction des trois propriétés

Registered deviations by $F(\theta)$ Equations in the prediction of the three properties

\begin{tabular}{l|c|c|c|c|c|c|c|c|c}
\hline \multicolumn{1}{c|}{ Propriétés } & \multicolumn{3}{|c|}{ Facteur acentrique } & \multicolumn{3}{c|}{ Tension superficielle } & \multicolumn{3}{c}{ Indice de corrélation } \\
\hline \multicolumn{1}{c|}{ Familles } & Intervalle & Nb. points & $\mathrm{AAD}(\%)$ & Intervalle & Nb. points & $\mathrm{AAD}(\%)$ & Intervalle & Nb. points & $\mathrm{AAD}(\%)$ \\
\hline$n$-paraffines & $\mathrm{C}_{5}-\mathrm{C}_{28}$ & 20 & 1,3 & $\mathrm{C}_{5}-\mathrm{C}_{36}$ & 18 & 0,8 & $\mathrm{C}_{4}-\mathrm{C}_{40}$ & 37 & 0,01 \\
$i$-paraffines & $\mathrm{C}_{5}-\mathrm{C}_{9}$ & 38 & 1,8 & $\mathrm{C}_{5}-\mathrm{C}_{11}$ & 36 & 1,8 & $\mathrm{C}_{5}-\mathrm{C}_{11}$ & 288 & 1,0 \\
Oléfines & $\mathrm{C}_{3}-\mathrm{C}_{20}$ & 45 & 5,8 & $\mathrm{C}_{3}-\mathrm{C}_{20}$ & 42 & 3,5 & $\mathrm{C}_{4}-\mathrm{C}_{40}$ & 194 & 1,5 \\
Alcynes & - & - & - & - & - & - & $\mathrm{C}_{4}-\mathrm{C}_{40}$ & 84 & 1,1 \\
Naphtènes & $\mathrm{C}_{3}-\mathrm{C}_{16}$ & 22 & 6,2 & $\mathrm{C}_{3}-\mathrm{C}_{16}$ & 24 & 2,8 & $\mathrm{C}_{4}-\mathrm{C}_{42}$ & 264 & 1,8 \\
Aromatiques & $\mathrm{C}_{7}-\mathrm{C}_{24}$ & 43 & 4,6 & $\mathrm{C}_{6}-\mathrm{C}_{24}$ & 42 & 2,8 & $\mathrm{C}_{6}-\mathrm{C}_{42}$ & 192 & 2,1 \\
$\mathrm{AAD}_{\text {moy }}(\%)$ & & 168 & 4,1 & & 162 & 2,5 & & 1059 & 1,5 \\
\hline
\end{tabular}




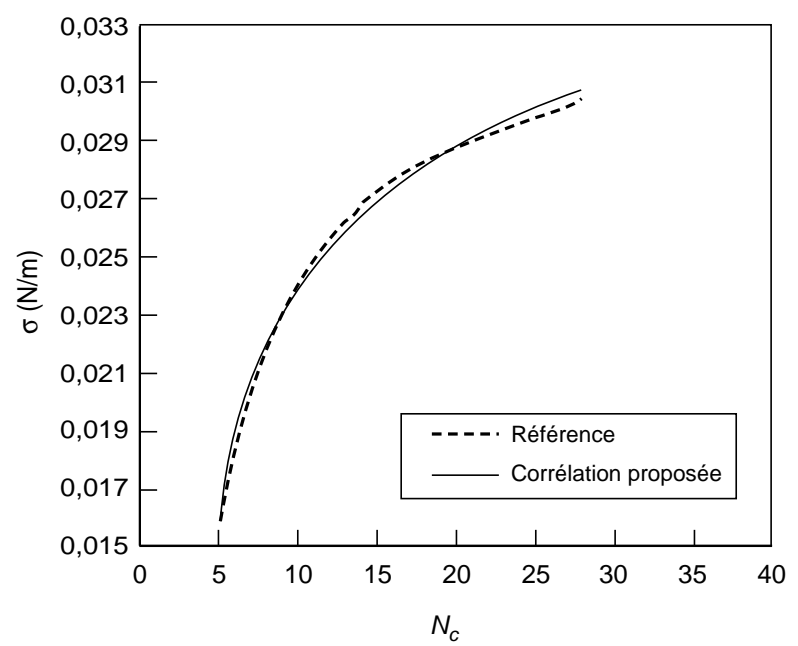

Figure 2

Tension superficielle en fonction du nombre d'atomes de carbone des $n$-paraffines.

Surface tensions versus n-paraffin carbon atoms.

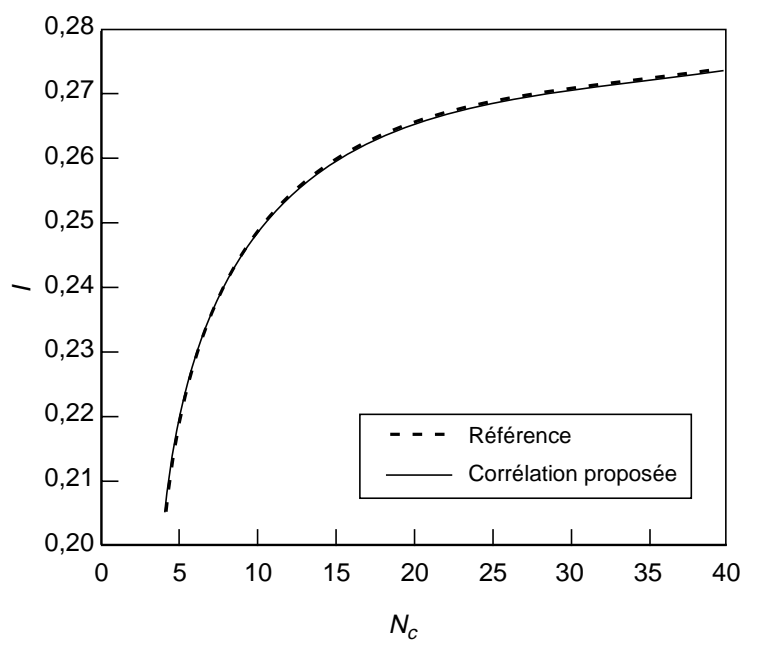

Figure 3

Indice de corrélation en fonction du nombre d'atomes de carbone des $n$-paraffines.

Refractive index parameters versus n-paraffin carbon atoms.

TABLEAU 3

Paramètres des corrélations de contribution de groupes sélectionnées

Equation parameters and group increment values for the selected equations

\begin{tabular}{|c|c|c|c|}
\hline & $I$ & $\sigma$ & $\omega$ \\
\hline Équations & 18 & 7 & 13 \\
\hline \multicolumn{4}{|c|}{ Paramètres } \\
\hline $\begin{array}{l}p \\
a \\
b \\
c \\
d \\
m \\
n\end{array}$ & $\begin{array}{c}9,97818 \mathrm{E}+00 \\
3,63704 \mathrm{E}+05 \\
-1,34807 \mathrm{E}+03 \\
4,47270 \mathrm{E}+06 \\
-1,19750 \mathrm{E}+03 \\
-1,36506 \mathrm{E}+00 \\
-1,65795 \mathrm{E}+01\end{array}$ & $\begin{array}{c}5,14717 \mathrm{E}-03 \\
-1,34872 \mathrm{E}+01 \\
1,99605 \mathrm{E}+00 \\
- \\
- \\
- \\
-\end{array}$ & $\begin{array}{c}3,64555 \mathrm{E}-01 \\
2,20846 \mathrm{E}-01 \\
-5,11881 \mathrm{E}-02 \\
3,49526 \mathrm{E}-01 \\
- \\
-4,26606 \mathrm{E}-01 \\
-\end{array}$ \\
\hline \multicolumn{4}{|c|}{ Contributions } \\
\hline $\begin{array}{l}-\mathrm{CH}_{3} \\
-\mathrm{CH}_{2}- \\
-\mathrm{CH}< \\
>\mathrm{C}< \\
=\mathrm{CH}_{2} \\
=\mathrm{CH}- \\
=\mathrm{C}< \\
=\mathrm{C}= \\
\equiv \mathrm{CH} \\
\equiv \mathrm{C}-\end{array}$ & $\begin{array}{c}-1,73997 \mathrm{E}-01 \\
5,23309 \mathrm{E}-01 \\
2,12424 \mathrm{E}+00 \\
3,91592 \mathrm{E}+00 \\
-1,64137 \mathrm{E}-01 \\
1,19728 \mathrm{E}+00 \\
3,08690 \mathrm{E}+00 \\
2,85119 \mathrm{E}+00 \\
-5,77688 \mathrm{E}-01 \\
1,66998 \mathrm{E}+00\end{array}$ & $\begin{array}{c}-3,60004 \mathrm{E}+00 \\
8,25009 \mathrm{E}+00 \\
2,00633 \mathrm{E}+01 \\
3,02211 \mathrm{E}+01 \\
-4,87426 \mathrm{E}+00 \\
1,11448 \mathrm{E}+01 \\
2,37906 \mathrm{E}+01 \\
1,98993 \mathrm{E}+01 \\
- \\
- \\
\end{array}$ & $\begin{array}{c}9,27784 \mathrm{E}-03 \\
6,39130 \mathrm{E}-03 \\
-1,51598 \mathrm{E}-04 \\
-9,22470 \mathrm{E}-03 \\
6,74079 \mathrm{E}-03 \\
6,30614 \mathrm{E}-03 \\
-1,16318 \mathrm{E}-03 \\
1,20166 \mathrm{E}-02 \\
- \\
- \\
\end{array}$ \\
\hline \multicolumn{4}{|c|}{ Termes correctifs tenant compte des effets de proximité des groupements $-\mathrm{CH}_{3}$} \\
\hline $\begin{array}{l}\mathrm{C}\left(\mathrm{CH}_{3}\right)_{3} \\
\mathrm{C}\left(\mathrm{CH}_{3}\right)_{2} \\
\mathrm{C}\left(\mathrm{CH}_{3}\right) \\
\mathrm{C}\left(\mathrm{CH}_{3}\right) \mathrm{C}\left(\mathrm{CH}_{3}\right) \\
\mathrm{C}\left(\mathrm{CH}_{3}\right) \mathrm{C}\left(\mathrm{CH}_{3}\right)_{2} \\
\mathrm{C}\left(\mathrm{CH}_{3}\right) \mathrm{C}\left(\mathrm{CH}_{3}\right)_{3} \\
\mathrm{C}\left(\mathrm{CH}_{3}\right)_{2} \mathrm{C}\left(\mathrm{CH}_{3}\right)_{2} \\
\mathrm{C}\left(\mathrm{CH}_{3}\right)_{2} \mathrm{C}\left(\mathrm{CH}_{3}\right)_{3}\end{array}$ & $\begin{array}{c}-1,61553 \mathrm{E}+00 \\
-9,42123 \mathrm{E}-01 \\
-2,88571 \mathrm{E}-01 \\
-4,18338 \mathrm{E}-02 \\
1,30256 \mathrm{E}-01 \\
-2,12830 \mathrm{E}-01 \\
3,32240 \mathrm{E}-01 \\
4,75670 \mathrm{E}-02\end{array}$ & $\begin{array}{c}-8,29792 \mathrm{E}+00 \\
-5,42904 \mathrm{E}+00 \\
-5,40113 \mathrm{E}-01 \\
-4,91464 \mathrm{E}-01 \\
3,48784 \mathrm{E}+00 \\
3,61863 \mathrm{E}+00 \\
6,67903 \mathrm{E}+00 \\
1,17050 \mathrm{E}+01\end{array}$ & $\begin{array}{c}7,67280 \mathrm{E}-04 \\
6,52209 \mathrm{E}-04 \\
-3,92719 \mathrm{E}-04 \\
4,68808 \mathrm{E}-04 \\
-7,46184 \mathrm{E}-04 \\
-9,98538 \mathrm{E}-04 \\
-1,86599 \mathrm{E}-03 \\
-2,71165 \mathrm{E}-03\end{array}$ \\
\hline
\end{tabular}


TABLEAU 3 (suite)

\begin{tabular}{|c|c|c|c|}
\hline \multicolumn{4}{|c|}{ Termes correctifs tenant compte des types de positions } \\
\hline $\begin{array}{l}\text { cis- } \\
\text { trans- }\end{array}$ & $\begin{array}{c}3,68380 \mathrm{E}-02 \\
-8,02791 \mathrm{E}-02\end{array}$ & $\begin{array}{l}-6,47076 \mathrm{E}-01 \\
-5,40387 \mathrm{E}+00\end{array}$ & $\begin{array}{l}-4,84188 \mathrm{E}-03 \\
-4,89108 \mathrm{E}-03\end{array}$ \\
\hline \multicolumn{4}{|c|}{ Termes correctifs tenant compte des effets du cycle } \\
\hline $\begin{array}{l}\text { Cycle à } 3 \text { atomes de carbone } \\
\text { Cycle à } 4 \text { atomes de carbone } \\
\text { Cycle à } 5 \text { atomes de carbone } \\
\text { Cycle à } 6 \text { atomes de carbone } \\
\text { Cycle à } 7 \text { atomes de carbone } \\
\text { Cycle à } 8 \text { atomes de carbone } \\
\text { Cycle à } 9 \text { atomes de carbone } \\
\text { Cycle à } 10 \text { atomes de carbone } \\
\text { Cycle à } 11 \text { atomes de carbone } \\
\text { Cycle à } 12 \text { atomes de carbone } \\
\text { Cycle à } 13 \text { atomes de carbone } \\
\text { Cycle à } 14 \text { atomes de carbone } \\
\text { Cycle à } 15 \text { atomes de carbone } \\
\text { Cycle à } 16 \text { atomes de carbone } \\
\text { Cycle à } 17 \text { atomes de carbone } \\
\text { Cycle à } 18 \text { atomes de carbone } \\
\text { Cycle à } 19 \text { atomes de carbone } \\
\text { Cycle à } 20 \text { atomes de carbone }\end{array}$ & $\begin{array}{c}-2,38277 \mathrm{E}+00 \\
-1,12038 \mathrm{E}+00 \\
-6,07989 \mathrm{E}-01 \\
2,77939 \mathrm{E}+00 \\
6,24364 \mathrm{E}+00 \\
1,46886 \mathrm{E}+01 \\
2,65938 \mathrm{E}+01 \\
3,84727 \mathrm{E}+01 \\
5,09464 \mathrm{E}+01 \\
5,35118 \mathrm{E}+01 \\
5,29898 \mathrm{E}+01 \\
4,93806 \mathrm{E}+01 \\
4,58045 \mathrm{E}+01 \\
3,93303 \mathrm{E}+01 \\
3,59399 \mathrm{E}+01 \\
3,26425 \mathrm{E}+01 \\
2,94533 \mathrm{E}+01 \\
2,39764 \mathrm{E}+01\end{array}$ & $\begin{array}{c}-1,05550 \mathrm{E}+01 \\
-1,15188 \mathrm{E}+01 \\
1,28618 \mathrm{E}+00 \\
5,31337 \mathrm{E}+00 \\
2,27374 \mathrm{E}+01 \\
- \\
- \\
- \\
- \\
- \\
- \\
- \\
- \\
- \\
- \\
- \\
- \\
-\end{array}$ & $\begin{array}{c}6,55265 \mathrm{E}-04 \\
1,71283 \mathrm{E}-03 \\
-8,52987 \mathrm{E}-04 \\
-6,27030 \mathrm{E}-03 \\
-9,45887 \mathrm{E}-03 \\
- \\
- \\
- \\
- \\
- \\
- \\
- \\
- \\
- \\
- \\
- \\
- \\
-\end{array}$ \\
\hline \multicolumn{4}{|c|}{ Termes correctifs tenant compte des effets du noyau aromatique } \\
\hline $\begin{array}{l}\text { Noyau aromatique } \\
\text { Substitution ortho- } \\
\text { Substitution méta- } \\
\text { Substitution para- } \\
\text { Substitution 1-2-3 } \\
\text { Substitution 1-2-4 } \\
\text { Substitution 1-3-5 } \\
\text { Substitution 1-2-5 } \\
\text { Substitution 1-2-6 } \\
\text { Substitution 1-3-4 } \\
\text { Substitution 1-2-4-5 } \\
\text { Substitution 1-2-3-4 } \\
\text { Substitution 1-2-3-5 } \\
\text { Substitution 1-2-3-4-5 }\end{array}$ & $\begin{array}{l}9,15520 \mathrm{E}+01 \\
1,50047 \mathrm{E}+01 \\
1,19657 \mathrm{E}+01 \\
1,36120 \mathrm{E}+01 \\
2,65694 \mathrm{E}+01 \\
2,01392 \mathrm{E}+01 \\
1,53820 \mathrm{E}+01 \\
6,33411 \mathrm{E}+01 \\
2,35815 \mathrm{E}+01 \\
2,61149 \mathrm{E}+01 \\
4,04895 \mathrm{E}+01 \\
5,49143 \mathrm{E}+01 \\
4,24739 \mathrm{E}+01 \\
6,94791 \mathrm{E}+01\end{array}$ & $\begin{array}{c}8,73425 \mathrm{E}+01 \\
4,94514 \mathrm{E}+00 \\
-2,65087 \mathrm{E}+01 \\
-4,10662 \mathrm{E}+01 \\
1,07349 \mathrm{E}+02 \\
-1,28212 \mathrm{E}+01 \\
-4,56259 \mathrm{E}+01 \\
- \\
- \\
- \\
1,03502 \mathrm{E}+02 \\
- \\
- \\
-\end{array}$ & $\begin{array}{c}-2,23559 \mathrm{E}-03 \\
1,21668 \mathrm{E}-04 \\
2,15093 \mathrm{E}-03 \\
5,27444 \mathrm{E}-03 \\
8,47769 \mathrm{E}-03 \\
1,38235 \mathrm{E}-02 \\
1,65129 \mathrm{E}-02 \\
- \\
- \\
- \\
2,01362 \mathrm{E}-02 \\
- \\
- \\
-\end{array}$ \\
\hline
\end{tabular}

TABLEAU 4

Comparaison entre les corrélations établies et celles de la littérature

Comparison between existing correlations and the proposed equations

\begin{tabular}{|c|c|c|c|c|c|c|}
\hline & $n$-paraffines & $i$-paraffines & Oléfines & Alcynes & Naphtènes & Aromatiques \\
\hline Facteur acentrique & & & AAD $(\%)$ & & & \\
\hline Corrélation proposée & 1,3 & 1,8 & 5,8 & - & 6,2 & 4,6 \\
\hline Riazi et El Sahhaf (1995) & 1,2 & - & - & - & - & - \\
\hline Constantinou (1995) & 2,6 & 2,8 & 6,8 & & 4,8 & 5,3 \\
\hline Tension superficielle & & & AAD (\%) & & & \\
\hline Corrélation proposée & 0,8 & 1,8 & 3,5 & - & 2,8 & 2,8 \\
\hline Riazi et El Sahhaf (1995) & 1,8 & - & - & - & - & - \\
\hline Indice de corrélation & & & $\operatorname{AAD}(\%)$ & & & \\
\hline Corrélation proposée & $\mathbf{0 , 0 1}$ & 1,0 & 1,5 & 1,1 & 1,8 & 2,1 \\
\hline Riazi et El Sahhaf (1995) & 0,01 & - & - & - & - & - \\
\hline
\end{tabular}


Afin d'illustrer la méthode établie, nous nous proposons de présenter ci-après des exemples de calcul des propriétés du 2,2,4,4-tétraméthylpentane (1) et du décylcyclohexane (2) dont les groupements sont représentés dans le tableau 5.

TABLEAU 5

Structure chimique des deux hydrocarbures

Chemical structure of the two hydrocarbons

\begin{tabular}{l|c|c}
\hline Principaux groupes & (1) & (2) \\
\hline $\mathrm{CH}_{3}$ & 6 & 1 \\
$\mathrm{CH}_{2}$ & 1 & 14 \\
$>\mathrm{CH}-$ & 0 & 1 \\
$>\mathrm{C}<$ & 2 & 0 \\
\hline Termes correctifs & & \\
\hline $\mathrm{C}\left(\mathrm{CH}_{3}\right)_{3}$ & 2 & 0 \\
Cycle à 6 atomes de carbone & 0 & 1 \\
\hline
\end{tabular}

Dans le tableau 6, les résultats obtenus sont comparés aux valeurs des tables ainsi qu'à d'autres méthodes de calcul recommandées dans la littérature.

\section{TABLEAU 6}

Propriétés des deux hydrocarbures

Properties of the two hydrocarbons

\begin{tabular}{c|l|c|c}
\hline$\theta$ & Méthodes & $\mathbf{( 1 )}$ & $\mathbf{( 2 )}$ \\
\hline \multirow{4}{*}{$I$} & Méthode proposée & 0,250 & 0,269 \\
& Tables (TRC, 1994) & 0,246 & 0,270 \\
& Déviation absolue (\%) & 1,6 & 0,4 \\
& Riazi et Daubert (1980) & 0,245 & 0,273 \\
& Déviation absolue (\%) & 0,4 & 1,1 \\
\hline \multirow{6}{*}{$\omega$} & Méthode proposée & 0,312 & 0,632 \\
& Tables (DIPPR, 1995) & 0,316 & 0,663 \\
& Déviation absolue (\%) & 1,3 & 4,7 \\
& Edmister (1958) & 0,344 & 0,577 \\
& Déviation absolue (\%) & 8,9 & 13,0 \\
& Constantinou et al. (1995) & 0,317 & 0,578 \\
& Déviation absolue (\%) & 0,3 & 12,8 \\
\hline \multirow{5}{*}{$\sigma$} & Méthode proposée & 0,020536 & 0,02967 \\
& Tables (DIPPR, 1995) & 0,019859 & 0,02863 \\
& Déviation absolue (\%) & 3,4 & 3,6 \\
& Brock et Bird (1955) & 0,02080 & 0,02627 \\
& Déviation absolue (\%) & 4,7 & 8,2 \\
\hline
\end{tabular}

\section{CONCLUSION}

De nouvelles corrélations de contribution de groupes ont été établies pour l'estimation des tensions superficielles, des facteurs acentriques et des indices de corrélation des hydrocarbures purs de type $n$-paraffines, isoparaffines, oléfines, alcynes, naphtènes et aromatiques. Les résultats obtenus ont montré que les corrélations établies sont fiables et présentent des écarts faibles par rapport aux données des tables. De plus, les corrélations proposées restent valables dans le cas des hydrocarbures lourds. Une extension de cette étude consisterait dans l'avenir à appliquer les équations proposées aux mélanges d'hydrocarbures et plus particulièrement aux fractions pétrolières.

\section{RÉFÉRENCES}

Benson, S.W., Cruickshank, F.R., Golden, D.M., Hougen, G.R., O'Neal, H.E., Rodgers, A.S., Shaw R. and Walsh R. (1969) Chemical Reviews, 69, 279-324.

Brock, J.P. et Bird, R.B. (1955) AIChE Journal, 1, 174.

Constantinou, L. et Gani, R. (1994) Aiche Journal, 40, 10, 16971710.

Constantinou, L., Gani, R. et O’Connell, J.P. (1995) Fluid Phase Equilibria, 103, 11-22.

DIPPR (1995) Design Institute of Chemical Engineers. Data Compilation of Pure Compound Properties.

Edmister, W.C. (1958) Applied Hydrocarbon Thermodynamics. Pet. Refiner, 37.

Joback, K.G. (1984) S.M. Thesis in Chemical Engineering, Massachuset Institute of Technology, Cambridge, 2.

Lydersen, A.L (1955) Coll. Eng. Univ. Wisconsin, Engineering Experimental Station Rept. 3.

TRC Tables (1994) Thermodynamic Data Base, version 1.3.

Riazi, M.R. et Daubert, T.E. (1980) Hydrocarbon Processing, March.

Riazi, M.R. et El Sahhaf, T.A. (1995) Ind. Eng. Chem. Res., 34, 11, 4145-4148.

Skander, N. et Chitour, C.E. (2002) Revue Oil \& Gas Science and Technologie, Revue de l'Institut Français du Pétrole, 57, 4, 369376.

Wuithier, P. (1972) Le pétrole: raffinage et génie chimique, tome 1, Éditions Technip.

Manuscrit définitif remis en mars 2004 